\title{
MARINE DIESELS WORKING CYCLE MONITORING ON THE BASE OF IMES GMBH PRESSURE SENSORS DATA
}

\author{
Stefan NEUMANN ${ }^{1}$, Roman VARBANETS ${ }^{2}$, Olena KYRYLASH ${ }^{2}$, \\ Vladyslav MAULEVYCH ${ }^{2}$, Oleksiy YERYGANOV ${ }^{2}$ \\ ${ }^{1}$ IMES GMBH, Germany \\ ${ }^{2}$ Odessa National Maritime University, Ukraine, e-mail: roman.varbanets@gmail.com
}

\begin{abstract}
The paper presents algorithms for marine diesel engines' working process monitoring designed by analyzing data obtained with IMES pressure sensors. Main features and properties of IMES sensors, used for 2- and 4-stroke modern marine diesel engines, are presented here. The pressure-time diagrams were taken as a basis for creating algorithms for marine diesel engines' working process monitoring. The task of preliminary calculation of top dead center position (TDC) of a cylinder was solved using polytropic compression equation. TDC position was calculated using the equation $\mathrm{P}^{\prime}=0$. Then algorithm for calculating the average indicator pressure and the indicator power of the cylinder has been determined. The CFD methods show how indicator valve channel influences the results of marine diesel engines' working process monitoring. With the example of compression curves in a cylinder, it's shown amplitude and phase distortions introduced into pressure measurements by the indicator valve channel. Such research work is important and actual in time due to the use of marine diesel portable performance analysis systems.
\end{abstract}

Keywords: diesel engine, cylinder pressure monitoring, top dead center, gas pressure sensor, Butterworth low-pass filter, mean indicated pressure, indicator valve channel

\section{INTRODUCTION}

Marine diesel engines' working process monitoring is carried out by analyzing diagrams of gas pressure in the working cylinders. For this needs IMES $\mathrm{GmbH}$ provides wide range of pressure sensors (fig.1) for 2-stroke low speed diesel engines and 4-stroke medium and high speed diesel engines [1]. The said sensors have reliable linear characteristics and got high stability for prolonged service and lifetime. High precision and low relative error of mentioned sensors have been proved by full-scale testing [2]. In this regard, they are widely used not only for conventional camshaft engines of MAN MC and Wartsila RTA types, but also in modern electronically controlled diesel engines of Wartsila RT-flex and MAN ME types.

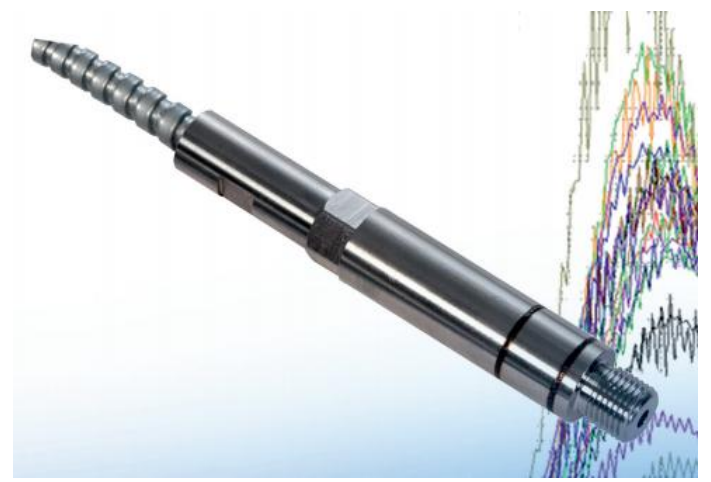

Fig. 1. IMES GmbH pressure sensor [1]
These types of diesels need constant controlling of cylinder gas pressure and main cycle parameters for the fuel combustion quality analyzing in the real time mode [3-5]. The strategy of fuel injection and exhaust valves controlling is based upon the analysis of gas pressure diagrams. The aim of such strategy is to maintain specified combustion characteristics, to reduce NOx emission or to reduce consumption, as well as to equalize mechanical and thermal loads between the cylinders.

Thus, it turns out to be critical for use of modern electronically controlled diesel engines to obtain high precision and stability of characteristics, that is provided by IMES pressure sensors in stationary performance analysis systems. Same tasks have to be solved in portable handheld system EPM-XP, which are produced also by IMES GmbH $[6,7]$ and used for periodic performance analysis (fig.2).

For both (stationary and portable) systems problem of data synchronization should be solved in a real time mode, i.e. data received from sensors has to be transferred from the time functions into crankshaft angle function:

$$
P(t, \mathrm{~ms}) \rightarrow P\left({ }^{\circ} \mathrm{CA}\right)
$$




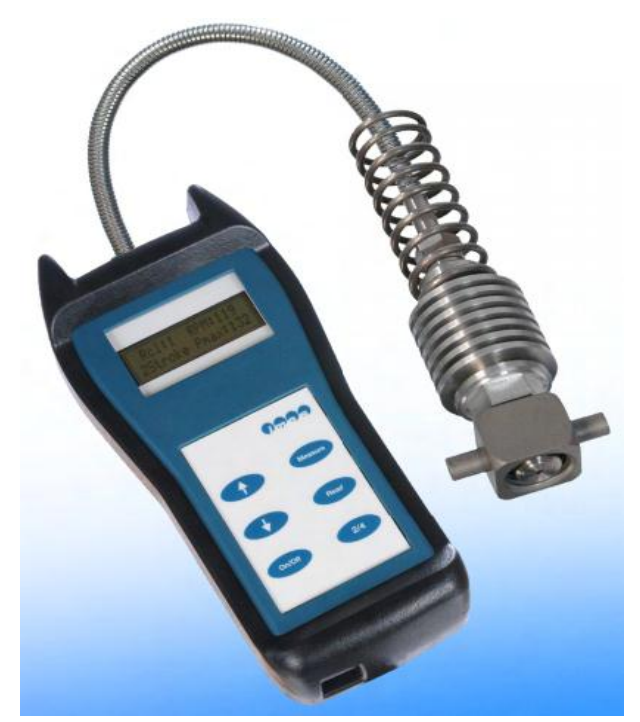

Fig. 2. IMES GmbH EPM-XP system with HTT cylinder pressure sensor including adaptor [7]

In most monitoring systems the working process of diesel engines, the pressure data are initially presented as pressure-time diagrams recorded with a time constant step. In practice, it is assumed that the minimum recording capacity should contain not less than 720 points per crankshaft revolution. In view of that, the main first task here is to determine TDC position of piston [8, 9].

For stationary performance analysis systems this task is solved mostly by instrumental method by means of pick-up sensors, installed on the flywheels of the engines [8]. In the case of portable systems, it is convenient to use the analytical procedure for TDC determination $[8,9]$.

Despite of simplicity and obviousness of the instrumental solution of the synchronization problem, it has a number of drawbacks:

- during the diesel engine working process unsteady phase error arises due to crankshaft twisting, rotation oscillations and microgaps of the crank mechanism;

- if the pressure sensor is installed at the end of the indicator valve channel, there will be phase and amplitude errors caused by the remote location of the sensor (as it will be shown further in the paper);

- in order to install pick-up sensors upon the engine flywheel, someone needs time and, moreover, it is necessary to stop the engine, that leads to certain costs and expenses.

The analytical method of TDC determination presented in this paper is free from these disadvantages. The method is based on the properties of polytropic compression in the working cylinder before the combustion. The proposed algorithm is based on the fact that when the piston passes TDC point, the rate of pressure growth is zero at a certain moment.

The importance of precise TDC determination for calculating mean indicated pressure (IMEP) and indicated cylinder power was described by different authors. In general, dependence of error while calculating indicated power upon the TDC calculating error is shown in the work by $\mathrm{M}$. Tazerout [10] in case of $1^{\circ} \mathrm{CA}$ error of TDC calculating, the error for mean indicated pressure is up to $9 \%$. In doctoral thesis [11], based on data analysis of the Sea Trials of MAN MC low speed diesel engines and mathematical modeling, is obtained the error in IMEP calculation equal 6-8 \% per $1{ }^{\circ} \mathrm{CA}$ error in TDC determination. In any case the influence of the error in TDC determination upon IMEP calculation is great. That is why in order to calculate IMEP and indicated power with engineering accuracy it is necessary to provide TDC determination with maximum error less than $0.25^{\circ} \mathrm{CA}$. It means that IMEP and then indicated power should be calculated by the curve $\mathrm{P}\left({ }^{\circ} \mathrm{CA}\right)$ by means of method of incrementing volumes, as will be shown later.

Thermodynamic methods of TDC calculation are proposed in $[10,12]$. They are also based, on the calculation of entropy for known pressure in the cylinder. Speaking about them, it can be noted that the presence of digital and analogue noises while measuring cylinder pressure can significantly reduce the accuracy of TDC determination. The numerical determination of the first, and especially the second, derivative of the pressure curve is meaningless without a correctly constructed digital filter.

\section{PERIODIC MONITORING OF CYLINDER PRESSURE WITH THE HELP OF EPM-XP ELECTRONIC INDICATOR}

Designed for periodic monitoring of cylinder pressure on diesel engines, the Electronic Indicator EPM-XP (fig. 3) can record cylinder pressure values (fig. 4) on a maximum of 20 cylinders (option: 160 cylinders) on two-stroke diesel engines operating at speeds of 40 to $300 \mathrm{rpm}$ and on fourstroke medium and high speed diesels with rated speeds from 200 to $1500 \mathrm{rpm}$ without using hardware pick-up sensors. Meantime synchronizing of diesel engines' performance diagrams without pick-up sensors is a task, which solution suggested in this article.

After acquisition, recorded data can be downloaded immediately to a PC or notebook via a USB connection. Recorded data can be simply processed by IMES' visualisation software. Transmitting the data by Internet makes them available for expert analysis and condition monitoring at a remote location. An optional software upgrade allows power calculation to assist cylinder balancing.

The EPM-XP cylinder pressure indicator is characterised by its high accuracy, reliability and ease of use. Central to the performance of EPM-XP is the proven accuracy, reliability, longevity and 
cost effectiveness of IMES' advanced type HTT pressure sensor employing TION thin film technology. They are affordable and their robustness and performance has been proven in many applications. As a result, standard effective life is more than 16,000 operating hours on both diesel and gas engines.

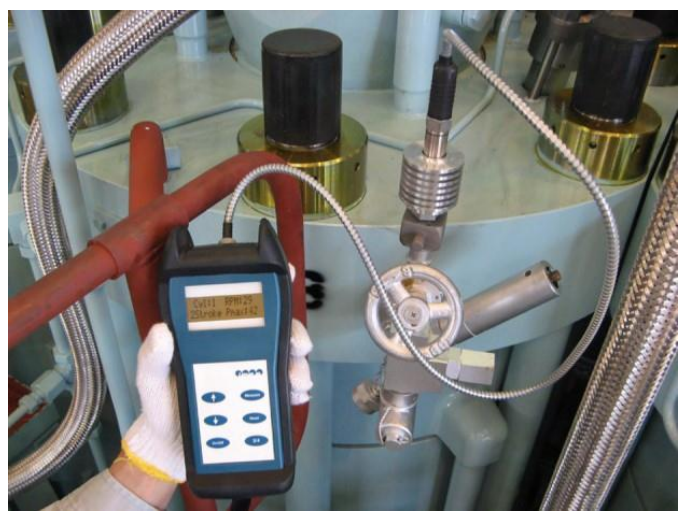

Fig. 3. EPM-XP application on MAN B\&W two-stroke diesel engine built under license by Kawasaki Heavy Industries [7]

IMES' visualisation and data processing software is used to process acquired values and to display the derived information. The resulting files can be transmitted by Internet to the engine builder and ship-owner for separate, independent evaluation

With the EPM-XP hardware dongle further evaluations can be activated. This enables to calculate IPOWER and IMEP by using a mathematical algorithm.
The advanced trending function is a useful tool to compare measurement data at the same engine output to find deviations in combustion process for preventive maintenance on engine. The analysis can be made by comparing the results to other measurements, mean values and configured limit lines (optimal range). Measurements made in long term indicate clearly the trends of the engine parameters, which will help to predict emerging failures.

Analytical solution of synchronization task is based on analysis of polytropic compression equation. For EPM-XP it's a necessity because of absence other sensors except of gas pressure sensor only. For stationary performance analysis systems numerical TDC determination will correct data received from pick-up sensors.

\section{DEFINITION OF TDC BY THE SOLUTION OF THE $P^{\prime}=0$ EQUATION}

The method is based on the fact that in case of combustion absence in the cylinder (fig. 5), the pressure change speed in TDC piston position equals to zero, minus TDC thermal loss angle that is connected with heat transfer to the cylinder walls:

$$
p_{\varphi-\delta \varphi_{T}}^{\prime}=p_{\Theta}^{\prime}=0
$$

taking into account TDC thermal loss angle in the top piston position, we get:

$$
\Theta=-\delta \varphi_{T} .
$$

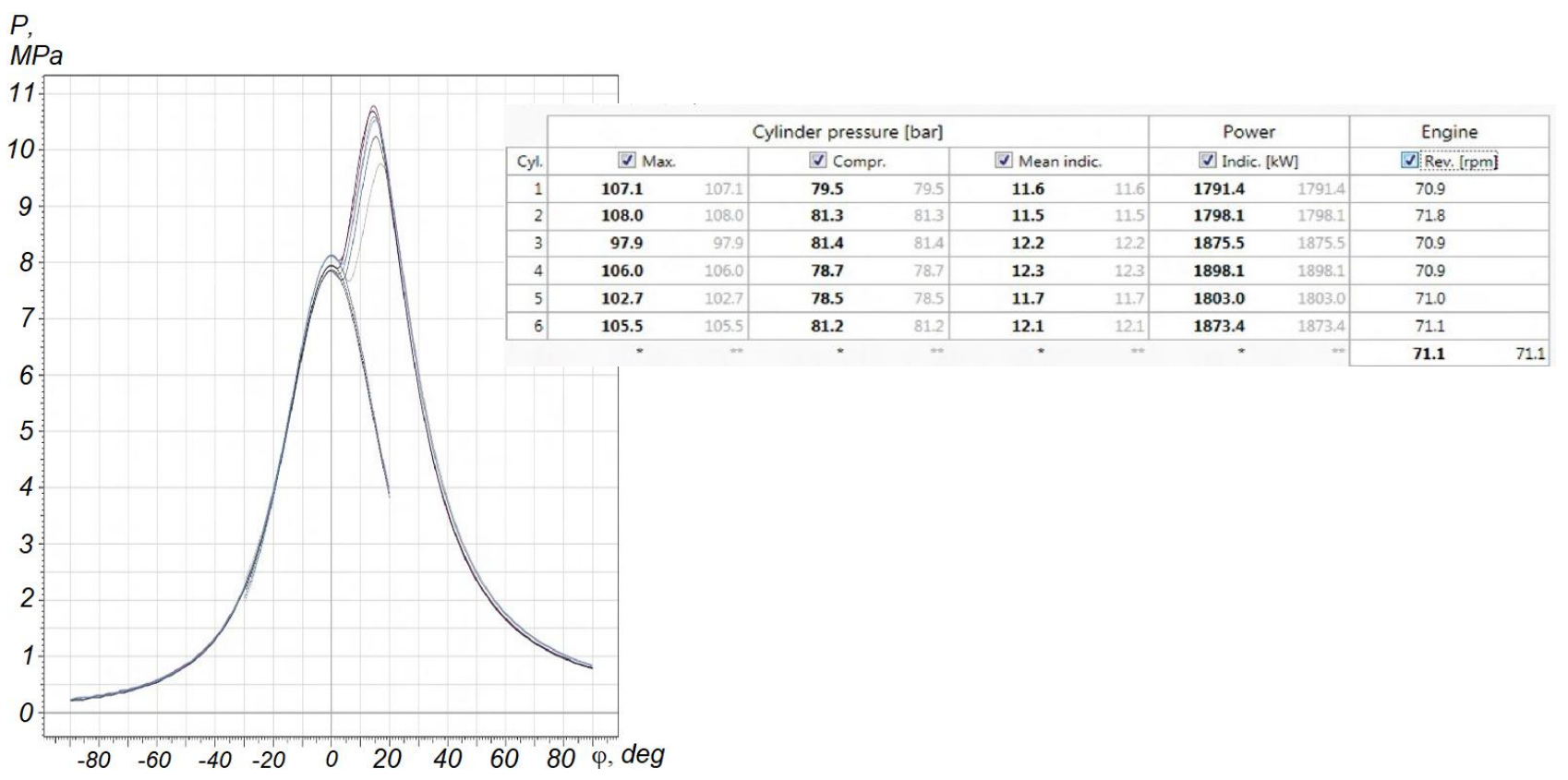

Fig. 4. Diesel engine performance analyses with the help of EPM-XP [7] 


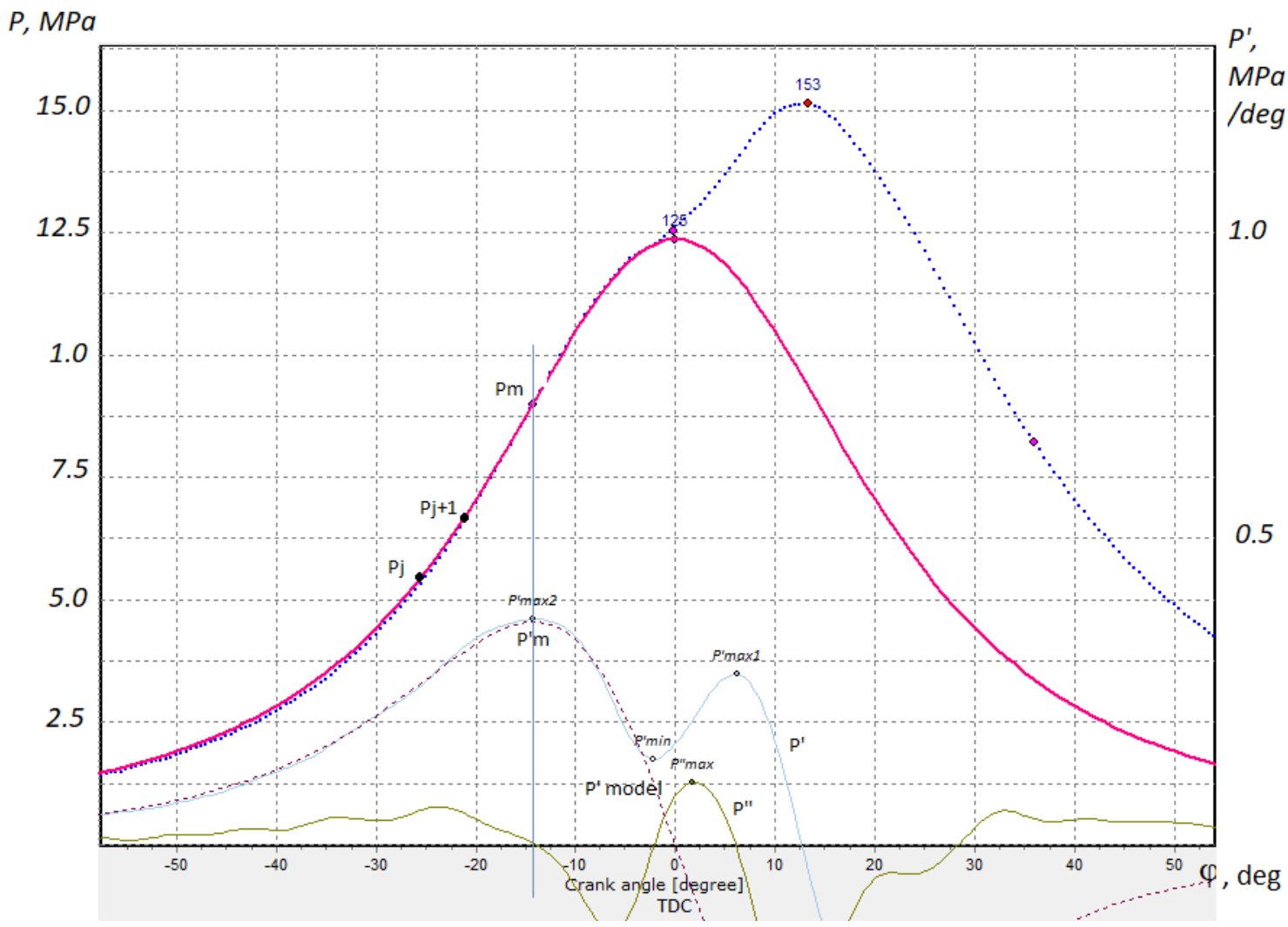

Fig. 5. Solving $P^{\prime}=0$ equation and TDC determination for YANMAR 6EY18ALW diesel engine

Then we can present pressure change speed at the compression section as follows:

$$
\frac{d P_{\text {comp }}}{d \Theta}=-P_{a} V_{a}^{n 1} n_{1} \frac{1}{V_{\Theta}^{n 1+1}} \cdot \frac{d V_{\Theta}}{d \Theta},
$$

where $P_{a}-$ pressure in the initial point of compression; $V_{\Theta}=V_{\varphi-\delta \varphi_{T}}-\quad$ cylinder volume, where:

$$
V_{\varphi}=V_{c}+0.5 V_{s}\left(\begin{array}{l}
1+1 / \lambda-\cos \varphi- \\
-1 / \lambda \sqrt{1-\lambda^{2} \sin ^{2} \varphi}
\end{array}\right)
$$

$V_{S}=V_{C}(\varepsilon-1)-$ the volume swept by the piston;

$V_{C}-$ compression chamber volume; $\varepsilon-$ compression ratio in cylinder;

$\lambda=R / L=S / 2 / L-$ the ratio of the crank radius to the length of the connecting rod.

By solving the (1) and (2) equations system we obtain the TDC coordinate (Fig. 5). Preliminary TDC coordinate can be obtained using values $P_{m}$ and $P_{m}^{\prime}$ - pressure and its angle derivative, as angle corresponding $P_{m}$ and $P_{m}^{\prime}$ is practically invariant from pressure and depends only upon geometrical sizes of crank mechanism, compression ratio and polytropic coefficient [13].

\section{DATA FILTRATION}

Despite high quality of IMES pressure sensors, their low relative error $(\sigma<1 \%)$ and low noise level, further numeric differentiation of the received indicator diagrams leads to practical impossibility of numeric analysis of curves $d P / d \varphi$. In this case determination of maximum $d P / d \varphi$ coordinates before the combustion, also will be carried out with rather big error (Fig. 6). It becomes obvious that it is necessary to apply the correct procedure for filtering the original data.

In this work the Butterworth digital low-pass filter [3] has been chosen because of its frequency characteristics are close to those of the ideal integrator, and are monotonic in the passband.

Frequency characteristics of $j$-th order Butterworth digital low-pass filter are:

$$
G(f)=\frac{G_{0}}{\sqrt{1+\left(f / f_{C}\right)^{2 j}}},
$$

where $G_{0}$ - zero-frequency gain coefficient; $f_{C}-$ the cutoff frequency (at which the amplitude is $3 \mathrm{~dB}$ [14]); $j$ - the filter number (order). 
The filter parameters can be selected/chosen using the recommendations [15]. Fig. 4 shows the $P(\varphi)$ and $P^{\prime}(\varphi)$ diagrams before the Butterworth low-pass filter was applied. Due to analog and digital noise' influence while recording $P(\varphi)$, determination of maximum $P^{\prime}(\varphi)$ coordinate will be made with a big error. The second $P^{\prime \prime}(\varphi)$ derivative analysis is just not possible in this case.

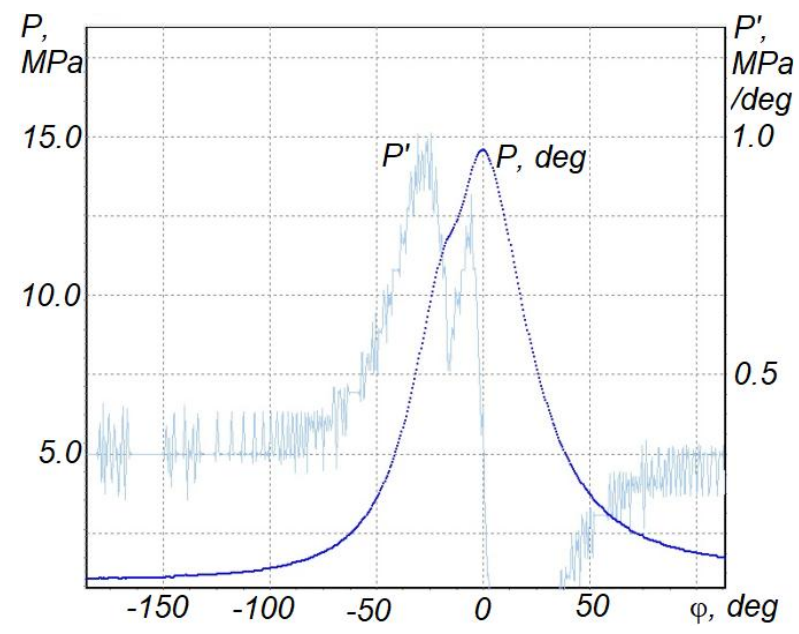

Fig. 6. Shifted cylinder pressure $P(\varphi)$ and its derivative $P^{\prime}(\varphi)$ before filtration

Application of the Butterworth low-pass filter makes it possible to analyze numerically the first $P^{\prime}(\varphi)$ and the second $P^{\prime \prime}(\varphi)$ derivatives (Fig. 7), as well as to determine the coordinate of the maximum compression stroke speed in the working cylinder (point $P^{\prime} m$ ).

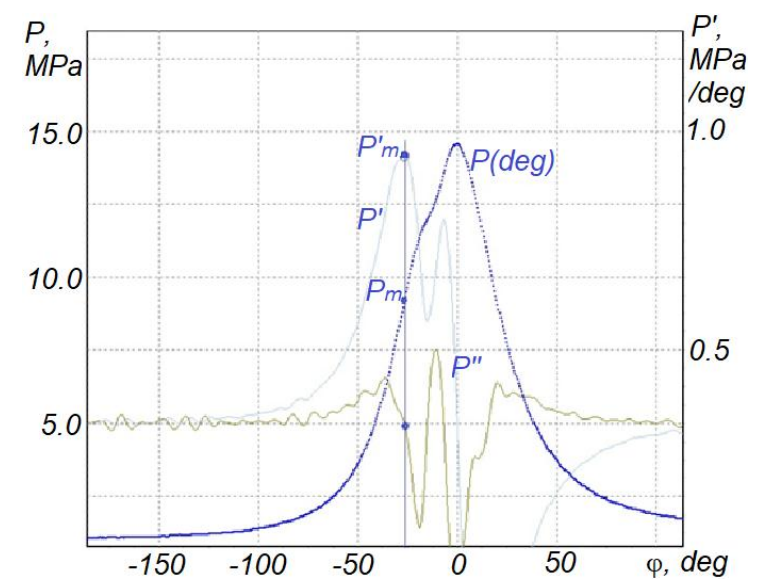

Fig. 7. Shifted cylinder pressure $\mathrm{P}(\varphi)$ and its derivatives $\mathrm{P}^{\prime}(\varphi)$ and $\mathrm{P}^{\prime \prime}(\varphi)$ after applying the Butterworth low pass filter

While recording indicator diagrams with the help of IMES pressure sensors (with relative error less than $1 \%$ and sampling step $\Delta \leq 0.5^{\circ} \mathrm{CA}$ ), the filter order $j=5.5 \div 7$ provided enough quality of high-frequency noise suppression on $P^{\prime}(\varphi)$ and $P^{\prime \prime}(\varphi)$ curves. Besides it made it possible to perform their further analysis for local extremum, which were later used to provide indicator diagrams synchronization, i.e. to transfer them from the functions of time into the crank angle degrees functions.

\section{CALCULATION OF CYCLE PARAMETERS}

Determination of the pressure, at which the combustion in the cylinder begins, is conducted by analyzing of the second-order cylinder gas pressure curve derivative. The beginning of combustion can be considered an inflection point on the pressure diagram, in which the value of the second derivative is maximal ( $P^{\prime \prime} \rightarrow \max$, fig. 8 ).

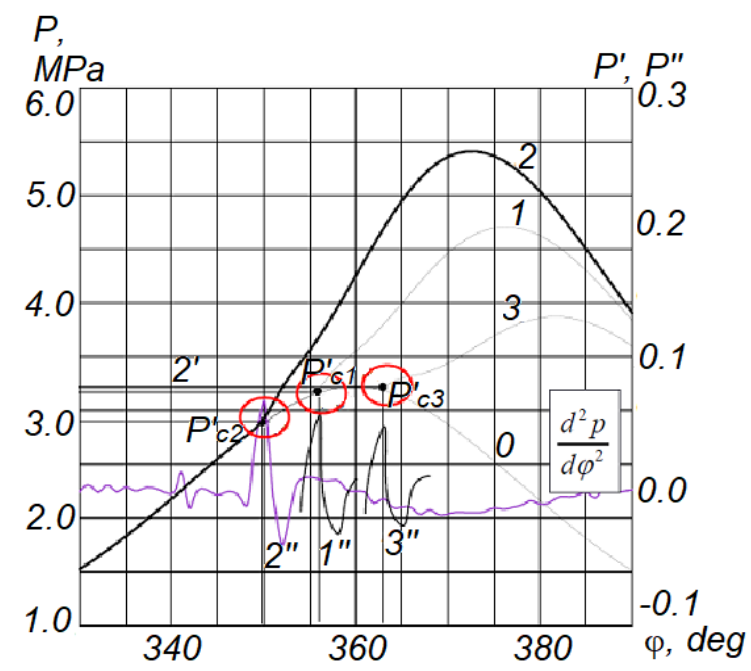

Fig. 8. Determination start of combustion at the different injection advance angles

The mean indicated pressure (IMEP) in the working cylinder is determined by the method of incrementing the volumes along the $P(\varphi)$ curve. IMEP is the specific work cycle, i.e. the cycle work per unit swept volume

$$
\operatorname{IMEP}=\frac{L}{V_{S}} .
$$

The difference in the work done on the extension stroke and spent on the compression stroke will be a useful work of the diesel cycle. Let's divide the indicator diagram $P(\varphi)$ into a number of elementary sections $\Delta l$. In this case, the work cycle will be equal to the amount of work at each site $\mathrm{L}=\Sigma \Delta l$, and the mean indicated pressure will be equal to:

$$
\mathrm{IMEP}=\frac{\sum \Delta l}{V_{s}} .
$$

The working volume of the cylinder is calculated by the formula: $V_{S}=\pi D^{2} / 4 S$, where $D$ - bore and $S$ - stroke of the piston [m]. The work done on each elementary section is (see Fig. 3):

$$
\Delta l_{j}=p_{k} \cdot V_{S k},
$$

where $p_{k}=\left(p_{j}-p_{j+1}\right) / 2$;

$$
V_{S k}=V_{j+1}-V_{j} \text {. }
$$


Thus, an expression for the mean indicated pressure of cycle could be written in the form of:

$$
\mathrm{IMEP}=\frac{1}{V_{S}} \sum_{j=1}^{m} \frac{p_{j}-p_{j+1}}{2}\left(V_{j+1}-V_{j}\right),
$$

where $m$ - the number of sections of the partition indicator diagram $P(\varphi)$.

For each angle of rotation of the crankshaft, the current volume of the cylinder $V_{j}$ is written as the expression (3).

Indicator power of the diesel is calculated by the formula

$$
\text { IPower }=c_{1} \cdot D^{2} \cdot S \cdot \mathrm{rpm} \cdot i \cdot \mathrm{IMEP}
$$

where rpm - revolution per minute; $i$ - number of cylinders; $c_{1}-$ the coefficient of the diesel engine stroke, taking into account the dimensions of the input values to bring the result to the SI system $\left(c_{1}=6.55\right.$ for 4 -stroke diesel engines, $c_{1}=13.1$ for 2-stroke diesel engines) [11].

\section{INFLUENCE OF THE INDICATOR VALVE CHANNEL ON THE RESULTS OF THE MARINE DIESELS PERFORMANCE ANALYSIS}

When monitoring serial low- and medium-speed marine diesel engines with portable monitoring systems, pressure sensors are installed on the indicator valve, the channel of which can be of considerable length. Gas-dynamic processes occurring in a long non-isothermal channel create methodological errors in determining the indicator parameters of the diesel engine. Therefore, the actual task is to reconstruct the undistorted gas pressure signal in the cylinder. To do this, the mathematical description of hydrodynamic and thermal processes occurring in the indicator valve channel and overpiston space is required. The authors of [16] investigated the processes in the channel of the indicator valve using onedimensional mathematical models of the flow of an ideal gas, and [17] - the flow of a viscous gas in a three-dimensional formulation using CFD (Computational Fluid Dynamics) methods. In both works the channel was considered separately from the overpiston space. The solution of the problem in such formulation requires the determination of the boundary conditions at the inlet of the channel and does not allow to take into account the complex relationship of processes in these elements. Combining elements into a single system "overpiston space - the indicator valve channel" will make it possible to model by the CFD methods interaction of the processes in this system with a high level of detail.

The authors investigated the effect of the indicator channel on the results of the indication of the marine diesel engine 6VDS48/42AL2 with the fuel feed switched off. The unsteady compressible laminar flow of air and heat transfer were modeled by the system of equations of continuity and conservation of momentum in the Navier-Stokes form and conservation of energy.

The periodic solution of the problem was sought by the finite volume method in the computational domain, including the air filled overpiston space and the indicator channel with the cavity at the sensor membrane.

The mathematical model is verified by the results of experimental studies of longitudinal air oscillations excited in a closed pipe by the motion of the piston, at a resonant frequency and far from it.

To obtain a periodic solution to the problem of air flow in the system "overpiston space - the indicator valve channel" (Fig. 9), the calculation was made for eight revolutions of the crankshaft. The results of the calculation showed a nonuniform distribution of the instantaneous pressure over the volume of the computational domain (Fig. 10, Fig. 11).

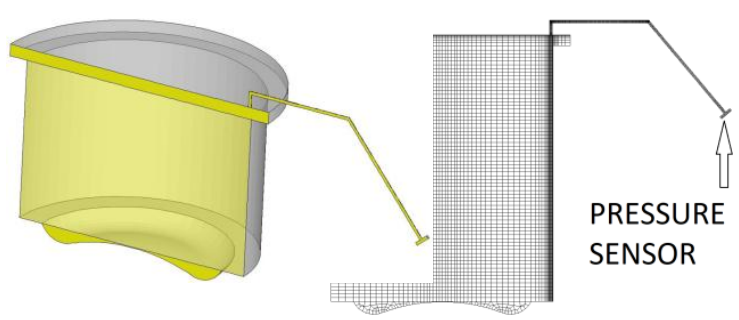

Fig. 9. Computational domain and grid at the position of the piston at the bottom dead center

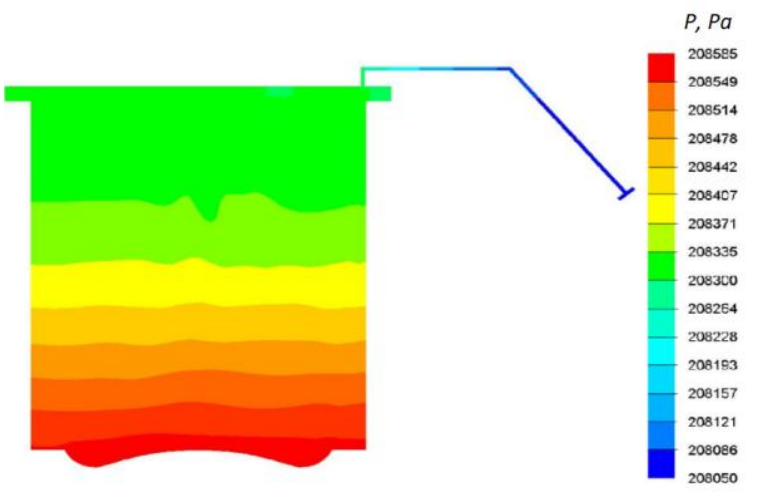

Fig. 10. Pressure in the cylinder and indicator valve channel in BDC

The instantaneous average air pressure in the cylinder and the instantaneous air pressure at the sensor mounting site were determined.

The calculation showed that at the end of the channel there is a phase delay of the signal by $0.5^{\circ}$ of the crankshaft rotation (Fig. 12, Tabl. 1). This is due to the spreading of the pressure pulse with the speed of sound. The maximum pressure in the cylinder $\left(p_{\max }\right)$ is offset from the top dead center of the piston and corresponds to the $178^{\circ}$ angle of rotation of the crankshaft due to the heat 


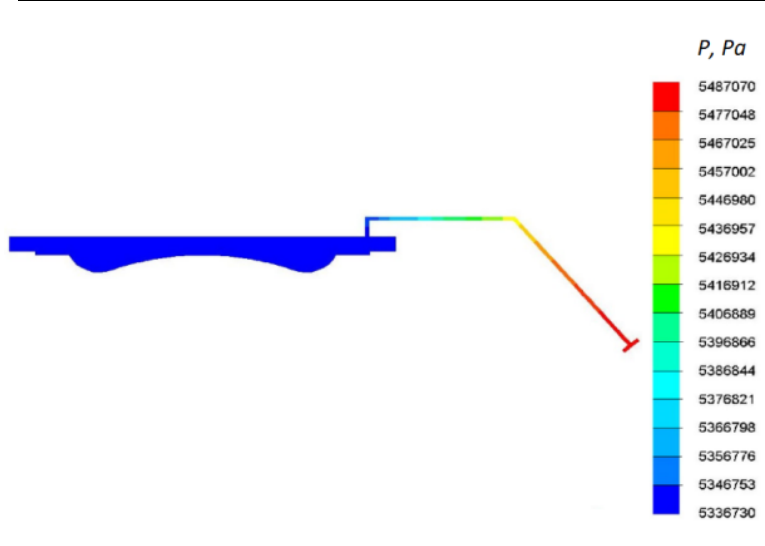

Fig. 11. Pressure in the cylinder and indicator valve channel in TDC

transfer to the cylinder walls. It is also evident that at the end of the channel the maximum pressure $\left(p_{\text {max.IVC }}\right)$ is $2.5 \%$ higher than in the cylinder (Fig. 12, Tabl. 1). This can be explained by the fact that when the piston is in the space nearly to the top dead center, the cause of the gas flow in the channel disappears, and the kinetic energy of the gas becomes potential, which causes an additional increase in pressure at the end of the channel.

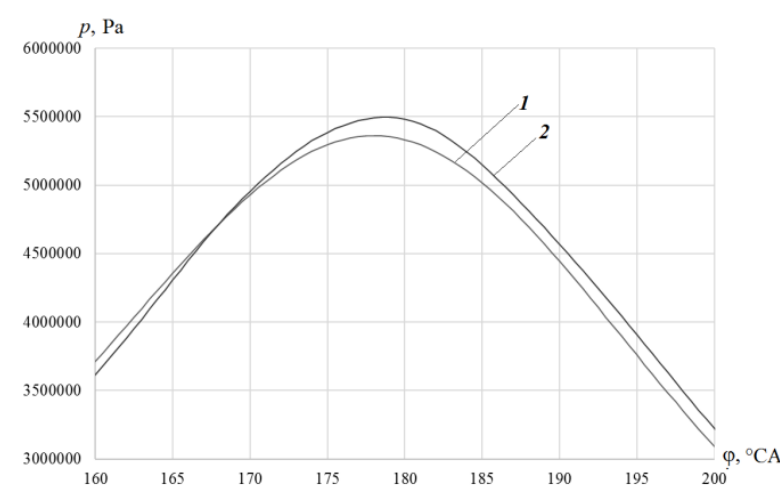

Fig. 12. Dependences of the average pressure in the cylinder (curve 1) and pressure at the end of the indicator valve channel (curve 2) from the angle of rotation of the crankshaft

Thus, the results of the calculation of the system "overpiston space - the indicator valve channel" showed the presence of a phase delay of the signal and an increase in the maximum air pressure at the end of the channel.

Table 1. The calculation results

\begin{tabular}{|l|c|}
\hline$p_{\text {max }}, \mathrm{Pa}$ & 5361448 \\
\hline$p_{\text {max.IVC }}, \mathrm{Pa}$ & 5497204 \\
\hline$\delta=\frac{p_{\max . I V C}-p_{\max }}{p_{\max }} \cdot 100 \%$ & 2,5 \\
\hline$\varphi_{\max },{ }^{\circ} \mathrm{CA}$ & 178 \\
\hline$\varphi_{\text {max.IVC }},{ }^{\circ} \mathrm{CA}$ & 178,5 \\
\hline
\end{tabular}

The observed distortion of the measured signal is due to the complex interaction of air flow processes with variable speed caused by the displacement of the piston, the propagation of pressure wave with the speed of sound and the propagation of disturbances from the previous cycle, and also heat transfer.

\section{CONCLUSIONS}

The TDC calculation using the equation $P^{\prime}=0$ and Butterworth low-pass filter solves the following problems:

- the errors in the determination of the TDC in connection with the torsional vibrations and the micro-backlash samples in the crank mechanism, and also with the crankshaft twisting under the load, which is relevant for cylinders far from the flywheel are taken into account;

- monitoring of the working process becomes operational and available for any diesel under load, without prior preparation;

- the calculation method for determining the TDC provides the practical accuracy required for determining IMEP and IPower.

When monitoring marine diesels with portable systems, phase and amplitude measurement errors arise due to the installation of the sensor at the end of the indicator valve channel. CFD calculation allows to take into account this effect.

\section{REFERENCES}

1. Neumann S. High temperature pressure sensor based on thin film strain gauges on stainless steel for continuous cylinder pressure control. CIMAC Congress 2001, Hamburg. Digest; 2001: 1 - 12.

2. Neumann S. Integrated cylinder pressure measurement for gas engine control. Combustion engines. 2011;3:146. http://www.combustionengines.eu/en/numbers/7/442

3. Wolfgang Krasa. Automatic optimization of diesel engine performance by loop control of cylinder pressure process. ICST Congress Hamburg, Sept.10, 2009

4. Wang YJ. et al. Fuel common rail injection system of rt-flex marine intelligent diesel engine and its simulation dynamic model. Advanced Materials Research, 2013;732-733:23-28.

5. MAN Diesel \& Turbo, MAN B\&W L60ME-C8-TII Project Guide Electronically Controlled Two-stroke Engines, 1st Edition. MAN Diesel \& Turbo, 2010.

6. CCM - combustion monitoring system: http://imes.de/cms.html

7. IMES launches EPM-peak pressure indicator: https://www.motorship.com/news101/shipsequipment/imes-launches-epm-peak-pressureindicator

8. Varbanets R, Karianskyi S, Rudenko S, Gritsuk I. et al. Improvement of diagnosing methods of the diesel engine functioning under operating conditions. SAE Technical Paper 2017-01-2218, 2017. https://doi.org/10.4271/2017-01-2218 
9. Varbanets R. Analyse of marine diesel engine performance / R. Varbanets, A. Karianskiy. Journal of Polish CIMAC. Energetic Aspects. - Gdansk: Faculty of Ocean Engineering and Ship Technology Gdansk University of Technology, 2012, 7(1):269275.

10. Tazerout M, Le Corre O, Rousseau S, TDC Determination in IC Engines Based on the Thermodynamic Analysis of the TemperatureEntropy Diagram, SAE Technical Paper 1999-011489, 1999. https://doi.org/10.4271/1999-01-1489

11. Varbanets RA. Diagnostic control of the working process of marine diesel engines in operation. Dissertation of the Doctor of Technical Sciences. Odessa, 2010. 314.

12. Staś M. An Universally Applicable Thermodynamic Method for T.D.C. Determination, SAE Technical Paper 2000-01-0561, 2000. https://doi.org/10.4271/2000-01-0561

13. Yeryganov $\mathrm{O}$, Varbanets R. Features of the fastest pressure growth point during compression stroke Diagnostyka 2018, 19(2):71-76 https://doi.org/10.29354/diag/89729

14. Laghari WM. Baloch MU, Mengal MA. Shah SJ. Performance Analysis of Analog Butterworth Low Pass Filter as Compared to Chebyshev Type-I Filter, Chebyshev Type-II Filter and Elliptical Filter. Circuits and Systems, 2014;5:209-216. http://dx.doi.org/10.4236/cs.2014.59023

15. Otnes Robert K, Enochson Loren. Applied Time Series Analysis. New York: Wiley, 1978: 449.

16. Malfunction diagnosis at marine diesel engines based on indicator cock pressure data / E. Oezatay, $\mathrm{Ph}$. Voegelin P. Obrecht Ch. Onder P. Fuchs W. Fuchs CIMAC Congress. - 2010; 166:22.

17. Turunen R. Cylinder pressure measurement via indicating cock / R. Turunen, O. Kaario G. Liljenfeldt CIMAC Congress. - 2007: 79-11.

Received 2018-11-23

Accepted 2019-02-18

Available online 2019-02-25

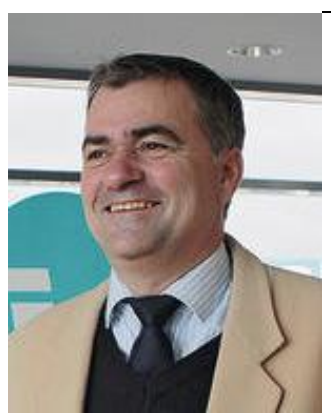

Stefan NEUMANN CEO IMES

$\mathrm{GmbH}$, is graduated engineer for energy and heat technology. $\mathrm{He}$ founded IMES $\mathrm{GmbH}$ in 1997. IMES is specialised in the field of combustion engine cylinder pressure and data acquisition systems. Its sensors, electronic indicators and combustion control and monitoring systems are employed on a wide range of diesel-, gas- and dual-fuel engines worldwide.

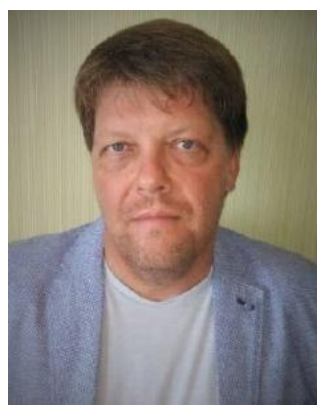

Roman VARBANETS Dr.Sc. defended his doctoral dissertation in Technical Sciences in 2010. Currently he is professor of marine engineering Head of "The Marine Power Plants and Technical Operation" department in Odessa National Maritime University. IMES GmbH consultant.

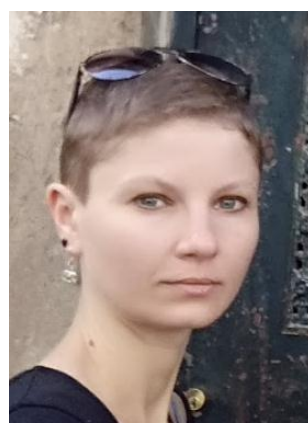

Olena KYRYLASH has received $\mathrm{PhD}$ in Technical Sciences in 2016. She worked as researcher in National Aerospace University "Kharkiv Aviation Institute", Department of Aircraft Engine Design.

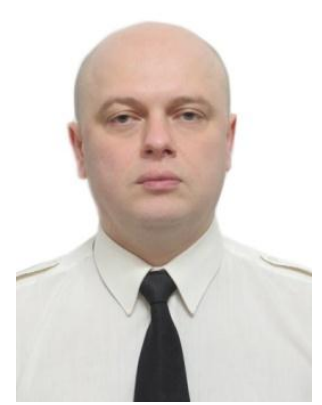

Oleksiy YERYGANOV has received his $\mathrm{PhD}$ in Technical Sciences in 2010. Currently he is assistant professor of "The Marine Power Plants and Technical Operation" Department in Odessa National Maritime University. He holds also Chief Engineer license for ships powered by internal combustion engines.

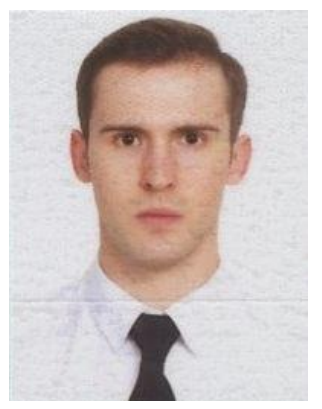

Vladyslav MAULEVYCH has completed the postgraduate study in 2018. At present he is lecturer of "The Marine Power Plants and Technical Operation" Department in Odessa National Maritime University. He holds also Second Engineer license for ships powered by internal combustion engines. 